\title{
Human Epididymis Protein 4 Expression in the Renal Cortex Tissue is an Independent Prognostic Factor in Patients with Renal Cell Carcinoma
}

Go Noguchi

Kanagawa Cancer Center

Ryosuke Jikuya

Yokohama City University Graduate School of Medicine

Yohei Miyagi

Kanagawa Cancer Center

Yoshiyasu Nakamura

Kanagawa cancer center

Shinji Ohtake

Yokohama City University Graduate School of Medicine

Yosuke Shibata

Kanagawa Cancer Center

Takahisa Suzuki

Kanagawa Cancer Center

Kimito Osaka

Kanagawa Cancer Center

Susumu Umemoto

Kanagawa Cancer Center

Yoji Nagashima

Tokyo Women's Medical University

Tomoyuki Yokose

Kanagawa Cancer Center

Takeshi Kishida

Kanagawa Cancer Center

Masahiro Yao

Yokohama City University Graduate School of Medicine

Noboru Nakaigawa ( $\nabla$ nakaigan@med.yokohama-cu.ac.jp )

Yokohama City University Graduate School of Medicine https://orcid.org/0000-0002-7228-3447

Research article 
Keywords: Renal cell carcinoma, Human Epididymis Protein 4, prognostic factor

Posted Date: August 26th, 2020

DOI: https://doi.org/10.21203/rs.3.rs-58033/v1

License: (c) (i) This work is licensed under a Creative Commons Attribution 4.0 International License. Read Full License 


\section{Abstract}

Background: Human epididymis protein 4 (HE4) has been indicated as a novel tumor marker for ovarian cancer diagnosis/monitoring and as a mediator of renal fibrosis. In this study, we investigated the potential role of HE4 in the pathogenesis of patients with renal cell carcinoma (RCC).

Methods: We retrospectively enrolled 249 patients with RCC who underwent nephrectomies at a single center between 1997 and 2010. HE4 expression was evaluated by immunohistochemistry and RNA in situ hybridization staining in both tumor and renal cortex tissue microarrays. The expression of HE4 was evaluated in line with the clinicopathologic features of each patient.

Results: HE4-positive staining was confirmed in 37 (15.0\%) tumor tissue microarrays and in 88 (38.3\%) cortex tissue microarrays. High expression of HE4 in the tumor was not associated with overall survival (OS), cancer-specific survival (CSS), or recurrence free survival (RFS). However, high expression of HE4 in the renal cortex was strongly associated with an increased risk of OS, CSS, and RFS (HR 3.608 (95\% confidence interval (Cl) 2.127-6.118); $p<0.001$, HR 5.759 (95\% Cl 2.801-11.841); $p<0.001$ and HR 3.309 (95\% $\mathrm{Cl} 1.751-6.252) ; p<0.001$, respectively). Multivariate analysis revealed that the Eastern Cooperative Oncology Scale of Performance Status (ECOG-PS), stage, pathological subtype, and high expression of HE4 in the renal cortex were independent risk factors for poor OS and that ECOG-PS, tumor size, stage, and high expression of HE4 in the renal cortex were independent risk factors for poor CSS.

Conclusions: High expression of HE4 in the renal cortex is an independent risk factor of OS and CSS in RCC. Further investigations are needed to elucidate the underlying mechanisms of HE4 in the renal cortex in the progression of renal cell carcinoma.

\section{Background}

Renal cell carcinoma (RCC) is the most common malignant tumor of the adult kidney, accounting for $3.8 \%$ of all new cancers [1]. Although the recent progress of screening has led to an increase in the rate of patients being diagnosed with RCC in early stages curable by treatment with radical surgery, approximately $15 \%$ of patients with RCC are diagnosed as having metastases at the initial presentation [2]. Resection of localized RCC by nephrectomy or partial nephrectomy is recommended as the only treatment for a complete cure; however, up to $30 \%$ of patients undergoing curative surgery will ultimately develop metastatic RCC $[3,4]$. Although molecular-targeted drugs, such as the tyrosine kinase inhibitors and inhibitors of the mammalian target of rapamycin, and immune-checkpoint inhibitors are currently available for the treatment of metastatic RCC, the effects of these drugs are limited. The median overall survival (OS) in metastatic disease is only 29 months [5]. The natural history of RCC is unpredictable, and patients with similar pathologic features may have very different clinical outcomes [6]. There is a great need to identify new prognostic biomarkers that, using surgical samples obtained during nephrectomy, can help predict prognosis in RCC. 
Human epididymis protein 4 (HE4) is a cancer biomarker that has recently garnered a great degree of interest from the research community. First identified in the epithelium of distal epididymis, HE4 was found to be frequently overexpressed in ovarian cancer (OC) and, to a lesser extent, in endometrioid tumors and lung cancer [7-9]. There are several studies suggesting that HE4 could be used as an independent biomarker for both the diagnosis and prognosis of $\mathrm{OC}[10,11]$. It has also been approved by the Food and Drug Administration as a biomarker to monitor patients with OC. While much is known about the predictive power of HE4 clinically, less has been reported regarding its molecular role in the progression of OC. James et al. reviewed HE4 signaling and suggested that HE4 promotes OC progression through pathways associated with cell proliferation, tumor growth, metastasis, chemoresistance, and steroid biosynthesis. They reported that results from numerous in vitro and in vivo studies provided compelling evidence that HE4 plays a role in cell proliferation and the promotion of tumorigenesis [12]. HE4 has also been shown to be a sensitive biomarker in renal fibrosis [13]. Wan et al reported higher serum concentrations of HE4 in patients with chronic kidney disease (CKD) and more severe renal fibrosis. Moreover, serum HE4 levels were found to be significantly increased in the early stages of CKD, and serum levels were also found to be correlated with the degree of renal fibrosis, suggesting that the upregulated expression of HE4 may present as one of the initial sign in the onset and progression of renal fibrosis [14]. However, the biological function of HE4 with regards to malignant tumors and renal fibrosis is not yet fully understood.

In the present study, we investigated the potential role of HE4 in the pathogenesis of patients with RCC. HE4 expression was evaluated by immunohistochemistry (IHC) in RCC tumors and renal cortex tissue. Furthermore, the association between HE4 expression and clinicopathological characteristics inlcuidng OS, cancer-specific survival (CSS), and recurrence free survival (RFS) rate in RCC patients was evaluated.

\section{Materials And Methods}

We retrospectively assessed the importance of HE4 expression in the pathogenesis of RCC. Clinicopathologic features and prognosis of patients with RCC were evaluated in association with HE4 expression.

\subsection{Patient Population}

The study protocol was reviewed and approved by the appropriate institutional ethics committees (2018Eki-35). Tissue samples from patients who underwent nephrectomies at Kanagawa cancer center hospital (Kanagawa, Japan) between 1997 and 2010 and were histologically diagnosed as RCC were used in this study. None of the patients underwent chemotherapy or radiotherapy prior to surgery. For each patient, the following clinicopathologic information was collected: age, gender, Eastern Cooperative Oncology Group performance status (ECOG-PS), estimated glomerular filtration rate (eGFR), histology, tumor size, pathological stage, and Fuhrman grade. All original Hematoxylin and Eosin (H\&E) slides were centrally reviewed by one experienced genitourinary pathologist (Y.N.) to obtain pathological features. 
Patients were staged using radiographic reports and postoperative pathologic data and were reassigned according to the 2016 World Health Organization classification.

\subsection{Tissue microarray}

Tissue microarray (TMA) was produced from the archives of formalin-fixed and paraffin-embedded (FFPE) tissue used for routine histopathologic diagnosis at the pathology department of Kanagawa Cancer Center. Briefly, an experienced genitourinary pathologist (Y.N.) centrally reviewed the H\&E stained slides and marked the areas to be punched out for TMA. Subsequently, we obtained FFPE tissue cores 2 $\mathrm{mm}$ in diameter corresponding to the marked areas on the H\&E slides with a manual tissue microarrayer (KIN-3 model, AZUMAYA Inc, Tokyo, Japan). For each case, representative two independent tumor areas and a non-neoplastic cortical area were processed to TMA.

\subsection{Immunohistochemical detection staining and evaluation}

HE4 protein IHC staining was performed on FFPE sections that were $4 \mu \mathrm{m}$-thick and mounted on Superfrost Plus slides cut from whole paraffin blocks (for 12 representative samples) and TMA (incorporated 249 patients) blocks. Deparaffinization and antigen retrieval were performed in Target Retrieval Solution, $\mathrm{pH} 9\left(120^{\circ} \mathrm{C}, 20 \mathrm{~min}\right)$. The rabbit monoclonal anti-HE4 IgG raised against recombinant human HE4 (EPR16658, Abcam, Cambride, UK) was used as a primary antibody at a 1:1000 dilution. The IHC procedure was performed using an autostainer HISTOSTAINER (Nichirei Biosciences Inc. Tokyo, Japan).

We determined the proportions of positively stained tumor cells or cortex cells in each section. The observations and measurements were conducted separately by two medical doctors (a pathologist and a urologist) without knowledge of patient outcome. In HE4 expression analysis, 15\% or more positivity was selected as a cut off values.

\subsection{In situ hybridization assay}

FFPE sections for in situ hybridization (ISH) were prepared as described for HE4 IHC. ISH was performed with RNAscope Probe-Hs-WFDC2 to detect HE4 mRNA and with RNAscope Negative Control Probe-DapB, both probes purchased form Advanced Cell Diagnostics: ACD (New York City, NY). Hybridization signals were visualized precisely with the RNAscope 2.5 HD kit (Red) (ACD) according to the manufacturer's instructions.

\subsection{Statistical analysis}

Student's t-test and Chi-square test were used to compare variables between subgroups of patients. OS, CSS, and RFS were calculated using the Kaplan-Meier method, and differences between groups were assessed by log rank tests. Cox proportional hazards model was used for univariate and multivariate analyses. All P values calculated were two-sided. A p-value $<0.05$ was considered to be statistically 
significant. Statistical analyses were performed using SPSS Software version 25.0 (SPSS Inc., Chicago, IL, USA).

\section{Results}

\section{Patient characteristics}

A total of 249 patients were enrolled in this study. The median follow-up period was 104 months. Table 1 shows the clinicopathologic features of the enrolled patients. A total of 68 patients $(27.3 \%)$ died during follow-up periods and the 5-year OS rate and the 5-year CSS rate were $82.5 \%$ and $86.1 \%$, respectively. Of the 213 patients who underwent curative surgery, 45 patients $(21.1 \%)$ had recurrent disease and the 5year RFS rate was $84.5 \%$.

\section{Evaluation of HE4 protein ICH with HE4 ISH}

We assessed HE4 expression by IHC and RNA ISH in tissue specimens. HE4 expression by RNA ISH was consistent with the results of IHC staining. Expression was primarily observed in uriniferous tubular cells. With regards to the IHC images of highly stained substances in the uriniferous tubules, it is possible that the HE4 protein is contained in the reservoir, as the tubular epithelium is positive in ISH (Fig.1).

\section{HE4 protein expression at the TMA}

We investigated HE4 protein expression in TMAs of 247 tumors and the corresponding 230 cortexes. HE4positive staining was confirmed in $37(15.0 \%)$ tumors and in 88 (38.3\%) cortexes. In the tumors, HE4positive staining was confirmed in $12.9 \%$ of all clear cell RCCs and in $27.0 \%$ of all non-clear cell RCCs $(p=0.026)$. In cortexes, HE4-positive staining was confirmed in $37.1 \%$ of all clear cell RCCs and in $45.5 \%$ of all non-clear cell RCCs $(p=0.358)$. HE4-positive staining was mainly located in the cytoplasm of tumor cells and uriniferous tubular cells at the cortex tissues (Fig. 2). The association between HE4 expression levels and clinicopathologic features were not detected in tumor TMAs. However, several correlations were presented at HE4 expression in the cortex TMA and clinicopathologic features (Table 2). Patients with a high expression of HE4 in the cortex were more likely to be older in age, have a lower eGFR, larger tumor size, higher pathological stage, and higher Furhman grade than the patients with low expression. No association was detected between HE4 expression in tumor TMAs and in cortex TMAs.

\section{High expression of HE4 is associated with cancer prognosis}

The high expression of HE4 in tumors was not associated with OS, CSS, or RFS (HR 1.379 (95\% Cl 0.7632.492); $p=0.287$, HR 0.505 (95\% Cl 0.181-1.410); $p=0.192$ and HR 0.468 (95\% Cl 0.167-1.310); $p=0.148$, respectively). However, Kaplan-Meier analyses indicated the high expression of HE4 at the cortex was associated with an increased risk of OS, CSS, and RFS (HR 3.608 (95\% Cl 2.127-6.118); $p<0.001, \mathrm{HR}$ 5.759 (95\% Cl 2.801-11.841); $p<0.001$ and HR 3.309 (95\% Cl 1.751-6.252); $p<0.001$, respectively) (Fig. 3 AC). The 5-year OS rate, CSS rate, and RFS rate were $69.4 \%, 76.8 \%$, and $72.9 \%$ in the high HE4 group and 93.5\%, $94.9 \%$, and $91.8 \%$ in the low HE4 group. Multivariate analysis revealed that the ECOG-PS, stage, 
pathological subtype, and high expression of HE4 were independent risk factors for poor OS (Table 3) and that ECOG-PS, tumor size, stage, and high expression of HE4 were independent risk factors for poor CSS (Table 4). For the RFS, ECOG-PS and pathological stage were independent risk factors; however, high expression of HE4 was not found to be an independent risk factor (Table 5.). However, patients who had recurrence of RCC with high HE4 expression $(n=24)$ had significantly worse OS and CSS after recurrence than patients with low HE4 expression ( $n=16)(\mathrm{HR} 2.777$ (95\% Cl 1.027-7.508); $p=0.044$ and HR 3.072 (95\% Cl 1.022-9.228); $p=0.046$, respectively).

We also performed a subgroup analysis for the expression of HE4 in OS by age, gender, ECOG-PS, eGFR, pathological size, pathological stage, Furhman grade, and pathological subtype (Fig. 4). The high expression of HE4 was a poor risk factor for OS, regardless of age, gender, tumor size, localized or metastatic, or Furhman grade. However, it was not significant risk factor for patients with a poor ECOGPS, low eGFR, or non-clear cell subtype.

\section{Discussion}

In this study, we analyzed HE4 expression in kidney tissue of patients with RCC. The results demonstrated that higher HE4 expression at the renal cortex tissue is an independent prognostic factor for patients with RCC. In particular, the 5-year CSS and 10-year CSS were $76.8 \%$ and $62.8 \%$, respectively, in the high expression group, whereas they were $94.9 \%$ and $92.1 \%$, respectively, in the low expression group, showing a strong hazard ratio (HR 5.759 (95\% Cl 2.801-11.841); $p<0.001$ ) (Fig. 2.B). This could be a very useful prognostic marker in clinical practice. To the best of our knowledge, this is the first study to investigate the impact of HE4 expression as a prognostic factor for RCC.

To date, there have only been two reports regarding the relationship between HE4 and RCC. Galgano et al. reported the expression of the HE4 gene and protein in a large series of various normal and malignant adult tissues by oligonucleotide microarray and TMA [7]. In this report, of the 26 clear cell RCC patients, only one (3.8\%) was weakly positive and all others were negative for HE4 staining. Of the 26 non-clear cell patients, $9(34.6 \%)$ were strongly positive, 6 (23.1\%) were weakly positive, and 11 (42.3\%) were negative for HE4 staining. Similarly, in our study, the expression rate of HE4 in tumor tissue was lower in clear cell RCC than in non-clear cell RCC $(12.9 \%$ vs $27.0 \%, p=0.026)$. Hertlein et al. compared the blood levels of HE4 in various cancers and benign disease and reported significantly higher levels in women with RCC compared to healthy individuals $(p<0.001)$. There were no significant differences found in blood levels between disease and healthy men $(p=0.613)$. They also showed that blood levels of HE4 increased with age, and that criteria differed between men and women, thus, they reported that age and gender specific reference ranges will have to be considered [15]. In our study, the expression of HE4 in the renal cortex could predict the prognosis of RCC regardless of gender and age (Fig. 3), thus it may be more convenient than measuring blood levels of HE4. Although the role of HE4 in RCC was limited in the above two reports, we confirmed that HE4 could be a tumor marker in RCC by observing the expression in the renal cortex. 
This study also aimed to address how the expression of HE4 in the renal cortex affected the poor prognosis of RCC patients. Although the exact physiological and pathological functions of HE4 are poorly understood for several cancers and renal fibrosis, Zhang et al. investigated the expression and mechanisms of HE4 in the pathogenesis of renal fibrosis by using the unilateral ureteral obstruction mouse model [16]. They found that hypoxia significantly increased HE4 expression in renal tubular epithelial cells. They suggested that hypoxia induces renal fibrosis by upregulating HE4 and activating the HIF-1 a/HE4/NF-KB signaling pathway. Based on their report, the mechanism of our results could be explained in that the kidney cortex tissue is relatively hypoxic due to being deprived of blood flow as a result of RCC, and that the expression of HE4 is subsequently increased. Under this hypothesis, it can be suggested that the high HE4 expression group included more patients with larger-sized, higher grade, and later stage tumors that require more blood supply. It can also be explained by the fact that a significant correlation with HE4 and poor prognosis was found only in the clear cell RCCs that are known for hyper vascularity (Fig. 3). This may also explain the high number of patients with renal dysfunction in the high HE4 group (Table 2). It is considered that renal fibrosis is enhanced due to an increase in the expression of HE4 in the cortex tissue, and renal dysfunction is appearing (Table 2). However, it is possible that HE4 expression was originally elevated in patients with renal dysfunction, and as a result, it is considered that there was no significant difference in patients with renal dysfunction as a result of subgroup analysis (Fig. 3). In this study, HE4 expression was a significant poor prognostic factor for OS and CSS, but not for RFS. This may be due to the small sample size, however patients with RCC reoccurrence and high HE4 expression levels had significantly worse OS and CSS than those with low HE4 expression levels.

Therefore, it was considered that the expression of HE4 may be associated with chemoresistance in RCC.

There are several limitations of this study. One of the major limitations is the limited sample size and the retrospectively evaluated oncological results. Furthermore, we did not investigate the function of HE4 in this study. However, this study shows that HE4 expression in the renal cortex is strongly correlated with renal cancer prognosis. In addition, this study indicates that HE4 protein is expressed and secreted in tubules, suggesting that it can be measured not only in the blood, but also in the urine. Further elucidation of mechanisms, confirmation of blood and urine levels, and prospective studies are needed.

In conclusion, this is the first study to demonstrate that high expression of HE4 in the renal cortex can be a great predictor of poor prognosis in RCC. This finding provides a novel independent predictor of clinical outcomes and may improve the management of patients with RCC with regards to prognostic stratification. Further investigation to elucidate underlying mechanisms is needed to validate these preliminary findings.

\section{List Of Abbreviations}

CKD: chronic kidney disease

CSS: cancer-specific survival

ECOG-PS: the eastern cooperative oncology group performance status 
eGFR: estimated glemerular filtration rate

FFPE: formalin-fixed and paraffin-embedded

H\&E: Hematoxylin and Eosin

HE4: human epididymis protein 4

IHC: Immunohistochemistry

ISH: In situ Hybridization

OC: ovarian cancer

OS: overall survival

RCC: renal cell carcinoma

RFS: recurrence free survival

TMA: tissue microarray

\section{Declarations}

\section{Ethics approval and consent to participate:}

This study was carried out in compliance with the Helsinki declaration and was approved by the Institutional Review Board (2018-Eki-35).

\section{Consent for publication:}

Not applicable.

\section{Availability of data and material:}

Due to ethical restrictions, the raw data underlying this paper is available upon request to the corresponding author.

\section{Competing interests:}

The authors declare that they have no competing interests.

\section{Funding:}

Not applicable.

\section{Authors' contributions:}


Data acquisition and Methodology: Go Noguchi, Ryosuke Jikuya, Yosuke Shibata, Shinji Ohtake, Takahisa Suzuki, Kimito Osaka, Susumu Umemoto

Histopathological evaluation: Yoji Nagashima, Tomoyuki Yokose

Formal analysis and investigation: Go Noguchi, Youhei Miyagi, Yoshiyasu Nakamura, Takeshi Kishida, Noboru Nakaigawa

Writing - original draft preparation: Go Noguchi

Writing - review and editing: Youhei Miyagi, Takeshi Kishida, Noboru Nakaigawa

All authors have read and approved the final manuscript.

\section{Acknowledgements:}

This study was supported by Kanagawa Cancer Center Hospital-Research Institute Joint Study.

We would like to thank Editage (www.editage.jp) for English language editing.

\section{References}

1. Moch H, Gasser T, Amin MB, Torhorst J, Sauter G, Mihatsch MJ: Prognostic utility of the recently recommended histologic classification and revised TNM staging system of renal cell carcinoma: a Swiss experience with 588 tumors. Cancer 2000, 89(3):604-614.

2. Siegel RL, Miller KD, Jemal A: Cancer statistics, 2018. CA Cancer J Clin 2018, 68(1):7-30.

3. Lam JS, Shvarts O, Leppert JT, Pantuck AJ, Figlin RA, Belldegrun AS: Postoperative surveillance protocol for patients with localized and locally advanced renal cell carcinoma based on a validated prognostic nomogram and risk group stratification system. J Urol 2005, 174(2):466-472; discussion 472; quiz 801.

4. Leibovich BC, Blute ML, Cheville JC, Lohse CM, Frank I, Kwon ED, Weaver AL, Parker AS, Zincke H: Prediction of progression after radical nephrectomy for patients with clear cell renal cell carcinoma. Cancer 2003, 97(7):1663-1671.

5. Motzer RJ, Hutson TE, Cella D, Reeves J, Hawkins R, Guo J, Nathan P, Staehler M, de Souza P, Merchan JR et al: Pazopanib versus sunitinib in metastatic renal-cell carcinoma. N Engl J Med 2013, 369(8):722-731.

6. Sun M, Shariat SF, Cheng C, Ficarra V, Murai M, Oudard S, Pantuck AJ, Zigeuner R, Karakiewicz PI: Prognostic Factors and Predictive Models in Renal Cell Carcinoma: A Contemporary Review. European Urology 2011, 60(4):644-661. 
7. Galgano MT, Hampton GM, Frierson HF, Jr.: Comprehensive analysis of HE4 expression in normal and malignant human tissues. Mod Pathol 2006, 19(6):847-853.

8. Yan L, Hu ZD: Diagnostic accuracy of human epididymis secretory protein $\mathbf{4}$ for lung cancer: a systematic review and meta-analysis. J Thorac Dis 2019, 11(7):2737-2744.

9. Mutz-Dehbalaie I, Egle D, Fessler S, Hubalek M, Fiegl H, Marth C, Widschwendter A: HE4 is an independent prognostic marker in endometrial cancer patients. Gynecol Onco/ 2012, 126(2):186-191.

10. Hellstrom I, Raycraft J, Hayden-Ledbetter M, Ledbetter JA, Schummer M, McIntosh M, Drescher C, Urban N, Hellstrom KE: The HE4 (WFDC2) protein is a biomarker for ovarian carcinoma. Cancer Res 2003, 63(13):3695-3700.

11. Ferraro S, Panteghini M: Making new biomarkers a reality: the case of serum human epididymis protein 4. Clin Chem Lab Med 2019, 57(9):1284-1294.

12. James NE, Chichester C, Ribeiro JR: Beyond the Biomarker: Understanding the Diverse Roles of Human Epididymis Protein 4 in the Pathogenesis of Epithelial Ovarian Cancer. Frontiers in Oncology 2018, 8.

13. Chen $P$, Yang Q, Li X, Qin Y: Potential association between elevated serum human epididymis protein 4 and renal fibrosis: A systemic review and meta-analysis. Medicine (Baltimore) 2017, 96(36):e7824 .

14. Wan J, Wang Y, Cai G, Liang J, Yue C, Wang F, Song J, Wang J, Liu M, Luo J et al: Elevated serum concentrations of HE4 as a novel biomarker of disease severity and renal fibrosis in kidney disease. Oncotarget 2016, 7(42):67748-67759.

15. Hertlein L, Stieber P, Kirschenhofer A, Krocker K, Nagel D, Lenhard M, Burges A: Human epididymis protein 4 (HE4) in benign and malignant diseases. Clin Chem Lab Med 2012, 50(12):2181-2188.

16. Zhang L, Liu L, Bai M, Liu M, Wei L, Yang Z, Qian Q, Ning X, Sun S: Hypoxia-induced HE4 in tubular epithelial cells promotes extracellular matrix accumulation and renal fibrosis via NF-kappaB. FASEB $J 2019$.

\section{Tables}

Due to technical limitations, table $1,2,3,4,5$ is only available as a download in the Supplemental Files section.

\section{Figures}




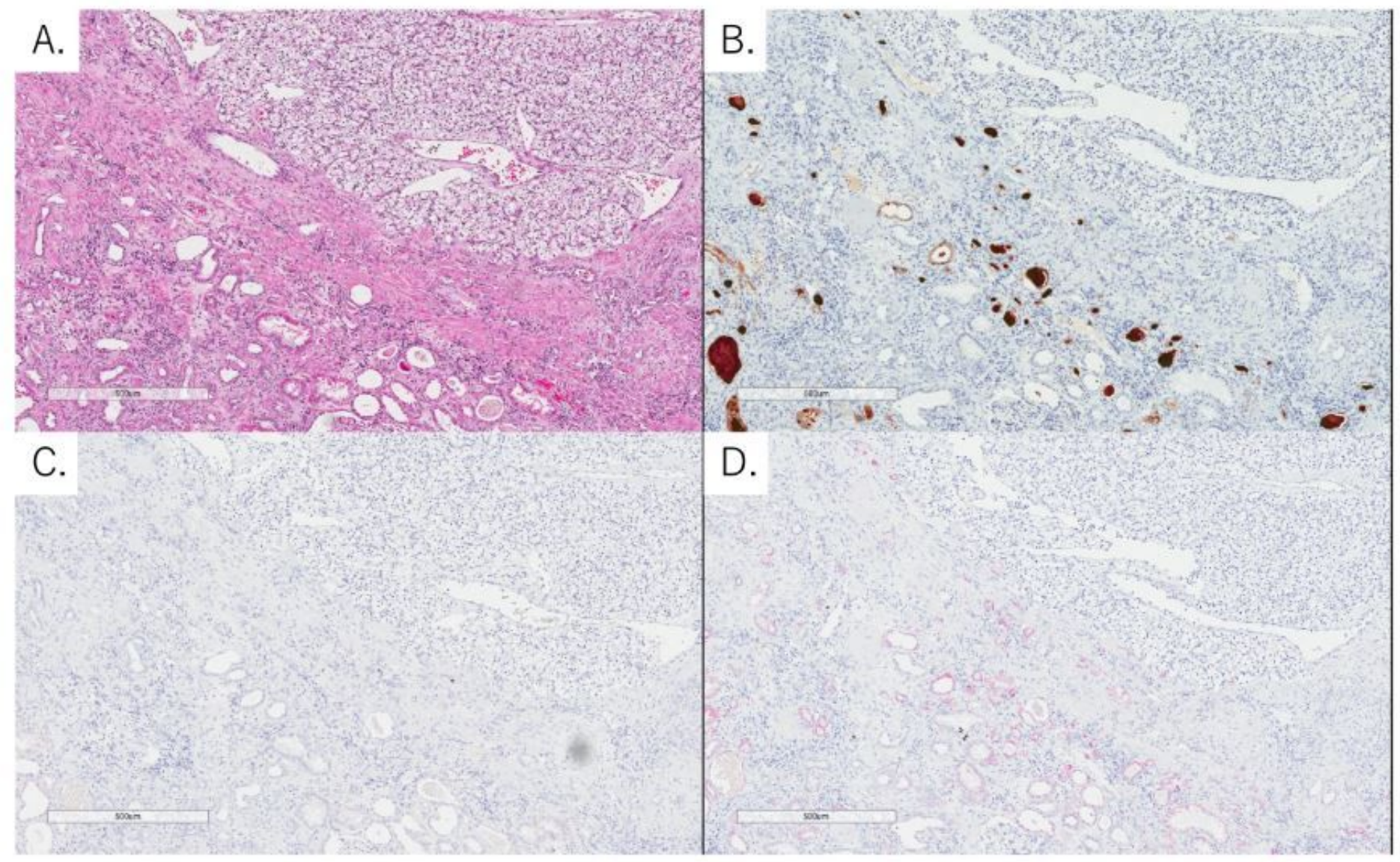

\section{Figure 1}

Comparison of HE, IHC, and ISH staining of HE4 expression in tissue specimens (A. HE staining, B. HE4 expression by IHC staining, C. RNA negative control, D. HE4 expression by RNA ISH staining). 

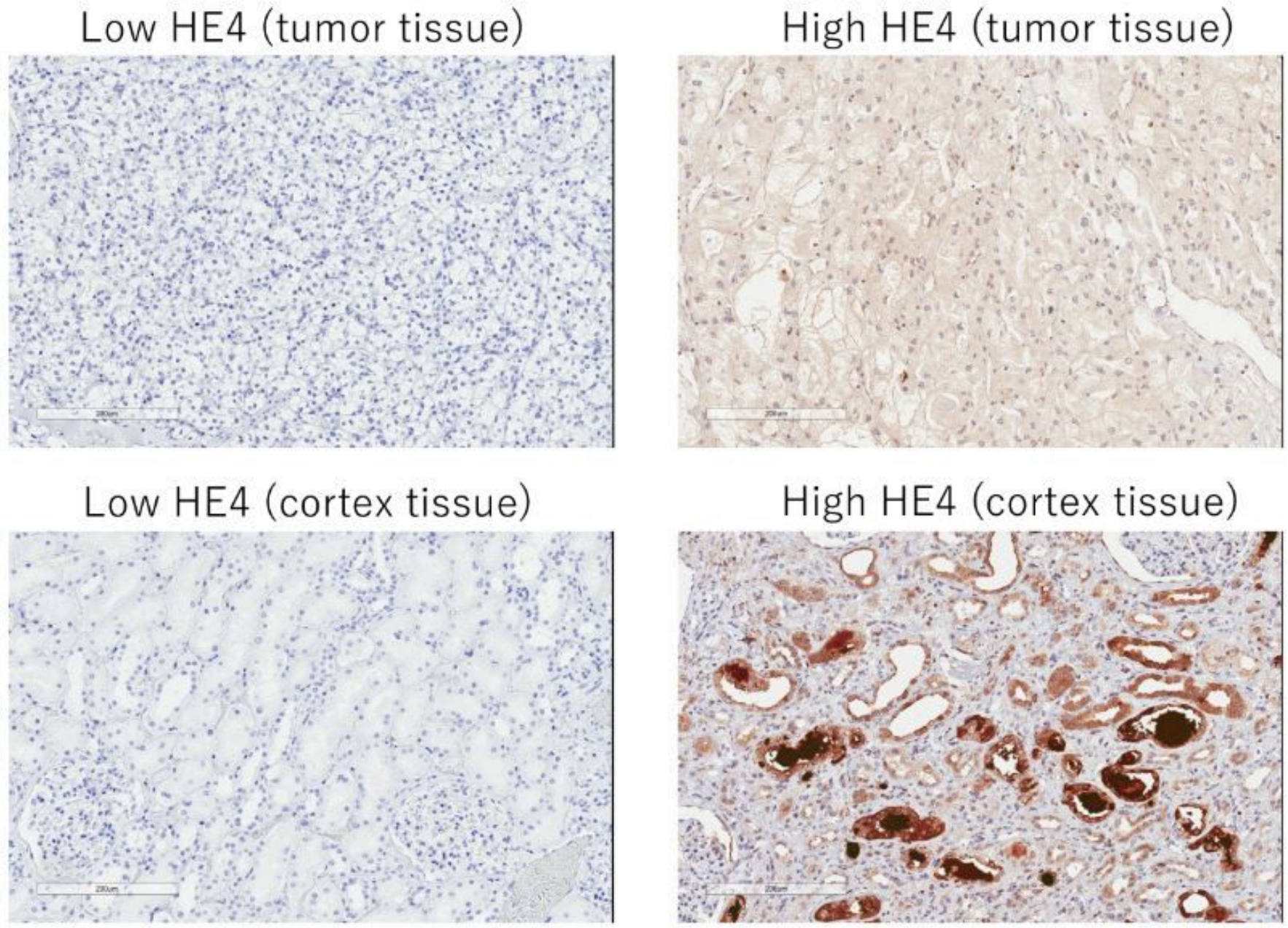

Figure 2

Expression of HE4 in tumors and renal cortex as per microarrays of RCC. In the tumor tissue, the cytoplasm of the cancer cells was lightly stained. In the cortex, tubular cells were strongly stained. A secretion with strong accumulation was also found inside the renal tubules. 
A.

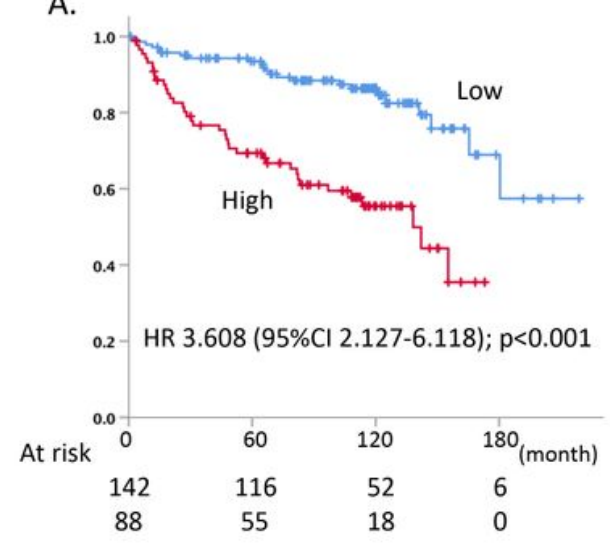

B.

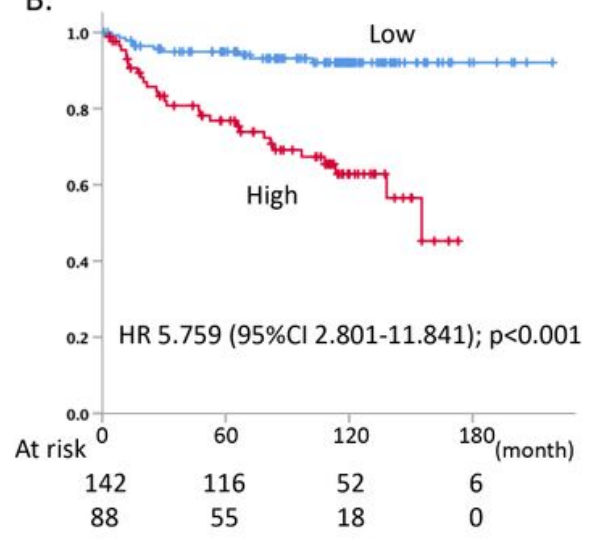

C.

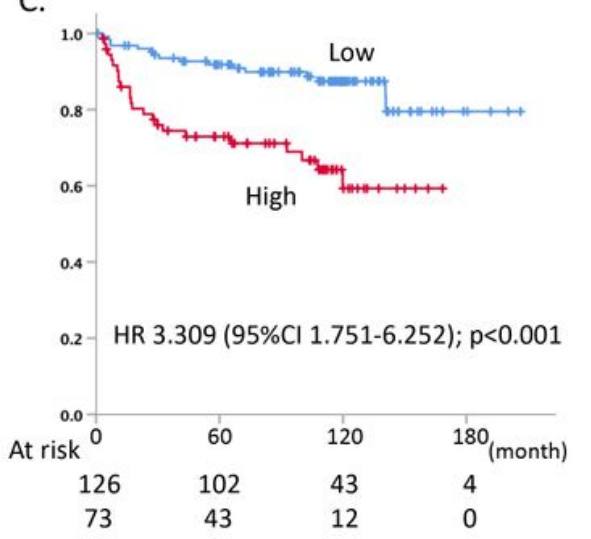

\section{Figure 3}

Kaplan-meier curves of OS (A), CSS (B), and RFS (C) divided by the expression of HE4s staining in the renal cortex.

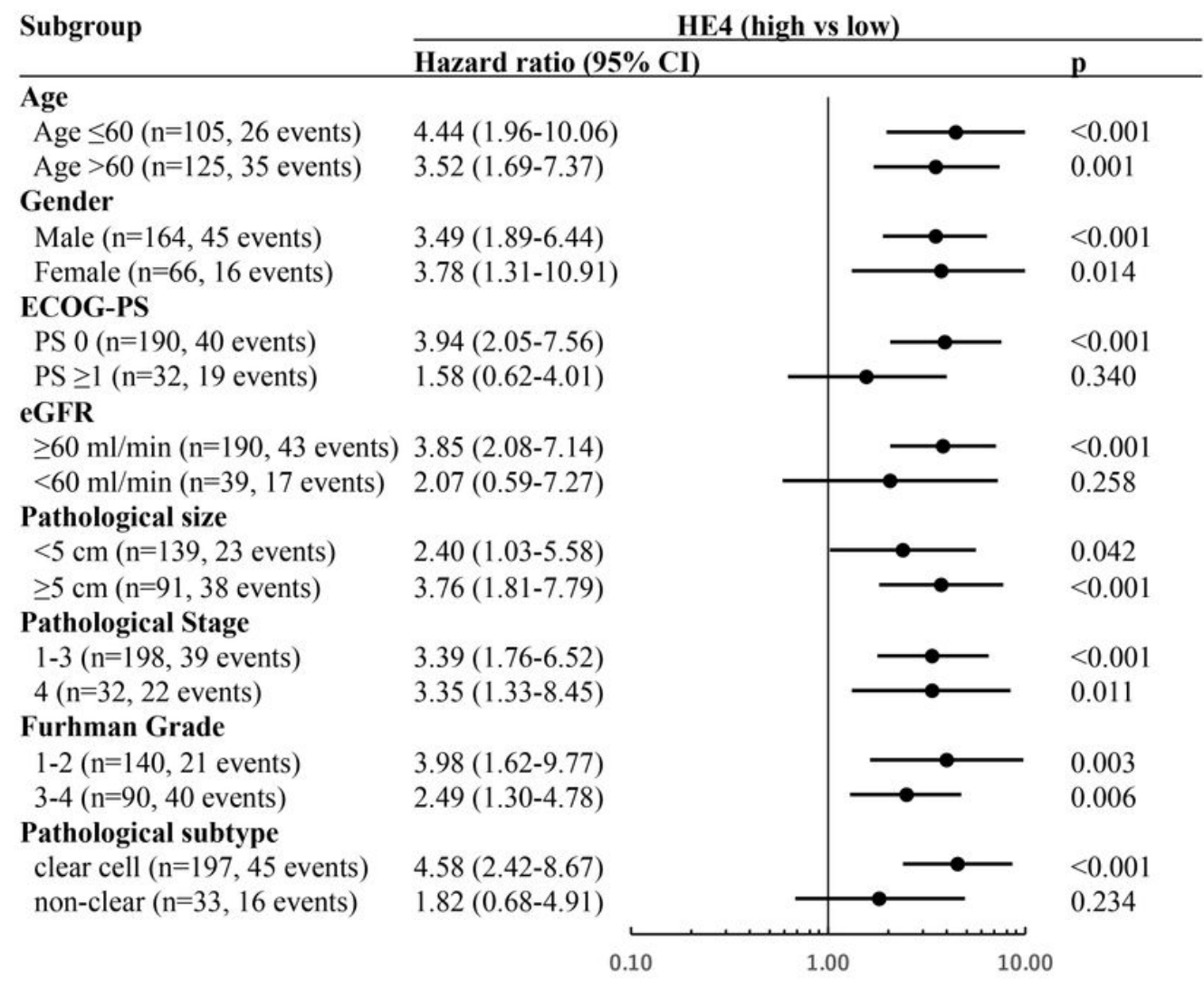


Figure 4

Hazard ratios for OS probabilities based on HE4 expression levels in patient subgroups.

\section{Supplementary Files}

This is a list of supplementary files associated with this preprint. Click to download.

- Table1.JPG

- Table2.JPG

- Table3.JPG

- Table4.JPG

- Table5.JPG 